\title{
Characterizing patient compliance over six months in remote digital trials of Parkinson's and Huntington disease
}

Shani Cohen ${ }^{1}$, Zeev Waks ${ }^{1 *}$ (D), Jordan J. Elm², Mark Forrest Gordon ${ }^{3}$, Igor D. Grachev ${ }^{4}$, Leehee Navon-Perry ${ }^{5}$, Shai Fine ${ }^{6}$, Iris Grossman ${ }^{7}$, Spyros Papapetropoulos ${ }^{8}$ and Juha-Matti Savola ${ }^{9}$

\begin{abstract}
Background: A growing number of clinical trials use various sensors and smartphone applications to collect data outside of the clinic or hospital, raising the question to what extent patients comply with the unique requirements of remote study protocols. Compliance is particularly important in conditions where patients are motorically and cognitively impaired. Here, we sought to understand patient compliance in digital trials of two such pathologies, Parkinson's disease (PD) and Huntington disease (HD).

Methods: Patient compliance was assessed in two remote, six-month clinical trials of PD ( $n=51$, Clinician Input Study funded by the Michael J. Fox Foundation for Parkinson's Research) and HD ( $n=17$, sponsored by Teva Pharmaceuticals). We monitored four compliance metrics specific to remote studies: smartphone app-based medication reporting, app-based symptoms reporting, the duration of smartwatch data streaming except while charging, and the performance of structured motor tasks at home.

Results: While compliance over time differed between the PD and HD studies, both studies maintained high compliance levels for their entire six month duration. None $(-1 \%)$ to a $30 \%$ reduction in compliance rate was registered for HD patients, and a reduction of 34 to 53\% was registered for the PD study. Both studies exhibited marked changes in compliance rates during the initial days of enrollment. Interestingly, daily smartwatch data streaming patterns were similar, peaking around noon, dropping sharply in the late evening hours around 8 pm, and having a mean of 8.6 daily streaming hours for the PD study and $10.5 \mathrm{~h}$ for the HD study. Individual patients tended to have either high or low compliance across all compliance metrics as measured by pairwise correlation. Encouragingly, predefined schedules and app-based reminders fulfilled their intended effect on the timing of medication intake reporting and performance of structured motor tasks at home.
\end{abstract}

Conclusions: Our findings suggest that maintaining compliance over long durations is feasible, promote the use of predefined app-based reminders, and highlight the importance of patient selection as highly compliant patients typically have a higher adherence rate across the different aspects of the protocol. Overall, these data can serve as a reference point for the design of upcoming remote digital studies.

Trial registration: Trials described in this study include a sub-study of the Open PRIDE-HD Huntington's disease study (TV7820-CNS-20016), which was registered on July 7th, 2015, sponsored by Teva Pharmaceuticals Ltd., and registered on Clinicaltrials.gov as NCT02494778 and EudraCT as 2015-000904-24.

Keywords: Remote clinical trials, Digital trials, Compliance, Wearables, Sensors, Smartphones, Parkinson's disease, Huntington disease

\footnotetext{
* Correspondence: zeev.waks@intel.com

'Advanced Analytics Department, Intel, 94 Em Hamoshavot Road, Petah

Tikva, Israel

Full list of author information is available at the end of the article
}

(c) The Author(s). 2018 Open Access This article is distributed under the terms of the Creative Commons Attribution 4.0 International License (http://creativecommons.org/licenses/by/4.0/), which permits unrestricted use, distribution, and reproduction in any medium, provided you give appropriate credit to the original author(s) and the source, provide a link to the Creative Commons license, and indicate if changes were made. The Creative Commons Public Domain Dedication waiver (http://creativecommons.org/publicdomain/zero/1.0/) applies to the data made available in this article, unless otherwise stated. 


\section{Background}

The widespread global use of smartphones and connected sensor devices, in parallel with their continuously increasing capabilities, has begun to impact the clinical trial ecosystem. Digital wearable and non-wearable devices containing electronic sensors such as accelerometers, gyroscopes, and photosensors can now track diverse biomarkers including heart rate, blood pressure, heart rate variability, lung function, and gait to various degrees of accuracy [1]. Complementarily, smartphone applications enable frequent and facilitated interaction with patients in the form of reminders, self-reporting of drug intake, or acquisition of electronic patient-reported outcomes [2].

Remote data collection via connected devices may provide added value to clinical trials [3]. Statistically, remote technologies increase data collection frequency, consequently providing insight on variability, requiring less extrapolation, and potentially increasing study power. These insights typically rely on signal processing and machine learning methods developed using sensor collected (big) data. Additionally, sensors, while not exclusively used in remote settings, are inherently objective compared to clinician scoring of disease status [4]. Home-based monitoring may also be more objective from the patient perspective owing to white cloak phenomena, muddying response in the clinic-setting [5]. From the trial management perspective, real-time monitoring may enable early identification of safety, operational, and compliance issues. Not surprisingly, applications for wearable technologies have already been demonstrated in a wide spectrum of disorders, including cardiovascular, respiratory, metabolic, psychiatric, and neurological disease [6]. Eventually, these innovations have the potential to evolve into regulatory-approved, clinical trial endpoints $[7,8]$.

Parkinson's disease (PD) and Huntington disease (HD) are both chronic, neurologically-based movement disorders. Motorically, PD is characterized by a diverse set of symptoms that present at successive stages of the disease, including slowing of gait, shuffling feet, reduced arm swing, freeze of gait, asymmetry, tremor, bradykinesia, and dyskinesia. In HD, the most notable movement impairment is chorea, which is often similar to PD dyskinesia but is generally stable in contrast to PD symptoms that may fluctuate throughout the day. Given their chronic nature, lack of adequate therapies, and unique motor aspects, PD and to a lesser extent HD have been the focus of many wearable studies in disease [7, 9].

Remote trials with smartphones and wearable devices have been conducted in movement disorders such as Parkinson's disease (PD) and Huntington disease (HD). Beyond a large collection of mostly short-duration studies aimed at quantifying symptoms [10], several efforts have examined longer-term, home-based monitoring in PD
[11-16]. Four notable examples of large, multi-month, PD efforts that assessed aspects of remote compliance are the Parkinson@Home study which quantified the daily duration in which a patient-worn smartwatch was streaming data over a period of up to 13 weeks $[13,17]$, the mPower and HopkinsPD studies which published results on smartphone app usage and performance of home-based tasks over six months $[14,15]$, and the SMART-PD study which evaluated the impact of a smartphone app on medication adherence over four months [16]. In contrast, in HD existing remote studies have primarily focused on feasibility or symptom quantification over several days $[9,18,19]$, as $\mathrm{HD}$ is an orphan disease which is naturally more challenging to recruit.

Despite its inherent advantages, remote patient-based data collection is prone to unique adoption and compliance issues $[6,20]$. Technical factors include the need for frequent device charging, ease of device operation, and the simplicity of the user interface. Burden factors unique to remote study protocols include, for example, the imposition of recurrent performance of home-based tasks or requirement for consistent reporting on symptoms and drug intake times. These issues are notable, as often a high number of patients dropout of studies prior to completion, even in 'traditional' trials [3]. Moreover, the effect of multi-month duration is important as patient compliance in clinical trials of chronic conditions is lower than in acute conditions [21]. Collectively, this highlights the importance of understanding compliance dynamics and patient preferences with respect to the requirements of remote digital trials. Motivated by above, we analyzed two six-month studies of neurological movement disorders to better understand patient compliance patterns in remote settings for four digital study protocol metrics.

\section{Methods}

Two independent clinical trials were analyzed: the Clinician Input Study in PD patients (CIS-PD) and the observational digital health sub-study within Study TV7820-CNS-20016 in HD patients (Open PRIDE-HD) (Table 1 and Table 2, respectively). The CIS-PD data used in the preparation of this article were obtained from the Michael J. Fox Foundation database. The total duration of each study was six months, and both studies used the Intel ${ }^{\circ}$ Pharma Analytics Platform for data collection, monitoring, and analysis [17, 22]. The mobile application interface was designed using User Interface/ User Experience (UI/UX) expert input to emphasize ease of use (Fig. 1).

\section{Parkinson's disease trial}

The CIS-PD trial was an observational study conducted in 4 US sites: Northwestern University, the University of 
Table 1 Characteristics of the PD and HD studies

\begin{tabular}{|c|c|c|}
\hline Parameter & CIS-PD study & Open PRIDE-HD sub-study \\
\hline Patient sample size & 51 & 17 \\
\hline Study duration & 6 months & 6 months \\
\hline Remote compliance metrics & $\begin{array}{l}\text { (1) Medication reporting } \\
\text { (2) Symptoms reporting } \\
\text { (3) Smartwatch data streaming }\end{array}$ & $\begin{array}{l}\text { (1) Medication reporting } \\
\text { (2) Symptoms reporting } \\
\text { (3) Smartwatch data streaming } \\
\text { (4) Structured motor assessments at home }\end{array}$ \\
\hline Sites & 4 sites in US & 11 sites across US, UK, Austria, Germany \\
\hline Devices & Apple Watch, iPhone & Pebble watch, iPhone \\
\hline App-based medication reporting & $\begin{array}{l}\text { The normal medication regimen } \\
\text { of the patient }\end{array}$ & $\begin{array}{l}\text { Pridopidine (interventional investigational } \\
\text { drug), twice per day per study protocol: } \\
\text { a morning dose between 7:00 and 12:00, } \\
\text { and an evening dose } 7 \text { to } 10 \mathrm{~h} \text { later }\end{array}$ \\
\hline App-based symptoms reporting & $\begin{array}{l}\text { Symptom severity three times } \\
\text { per day }\end{array}$ & $\begin{array}{l}\text { Chorea severity during the last five minutes, } \\
\text { once per day }\end{array}$ \\
\hline Wearing of smartwatch & $\begin{array}{l}\text { A minimum of } 12 \mathrm{~h} \text { per day } \\
\text { for } 25 \text { days per month }\end{array}$ & $\begin{array}{l}\text { Continuously throughout study duration, } \\
\text { preferably between 9:00-21:00 }\end{array}$ \\
\hline $\begin{array}{l}\text { Performance of structured motor } \\
\text { assessments at home }\end{array}$ & NA & Every other day, alternating mornings and evenings \\
\hline
\end{tabular}

Cincinnati, the University of Rochester, and the University of Alabama [23]. The study recruited 51 participants between June 2017 and August 2017, with 36 patients completing the entire study. Eligibility criteria included diagnosis of idiopathic PD, age 18 years old or older, Hoehn \& Yahr stage 1-3, informed consent, and lack of cognitive impairment that would preclude study participation. Study participants used their own iPhone smartphone and were provided an Apple Watch smartwatch containing the Intel ${ }^{\bullet}$ Pharma Analytics Platform application. In-clinic visits occurred at baseline, after 2 weeks, after 1 month, after 3 months, and 6 months after baseline, during which clinicians performed assessments and reviewed a dashboard that dynamically displayed data from the smartwatch and smartphone application. The study also had a remote component as described below.

We tracked the three protocol-defined metrics related to remote participation: (1) app-based medication reporting, (2) smartwatch data streaming, and (3) app-based daily symptoms reporting (Fig. 1). We define smartwatch data streaming as the hours in which the smartwatch was broadcasting accelerometer data, not including charging

Table 2 Demographics and disease status of the PD and HD patients. MDS-UPDR: Movement Disorder Society-Unified Parkinson's Disease Rating Scale. UHDRS-TMS: Unified Huntington Disease Rating Scale Total Motor Score

\begin{tabular}{lll}
\hline Demographics & CIS-PD study & Open PRIDE-HD sub-study \\
\hline Total patients & 51 & 17 \\
Gender: males & $29(57 \%)$ & $9(53 \%)$ \\
Ethnicity: Caucasian & $45(88 \%)$ & $16(94 \%)$ \\
Ethnicity: Hispanic & $1(2 \%)$ & $1(16 \%)$ \\
Age (mean \pm s.d.) & $62 \pm 11$ & $51 \pm 12$ \\
Years since symptom onset (mean \pm s.d.) & $9 \pm 5$ & NA \\
Years since diagnosis onset (mean \pm s.d.) & $7 \pm 5$ & NA \\
PD: Hoehn \& Yahr (mean \pm s.d.) & $2 \pm 0.42$ & NA \\
PD: MDS-UPDRS Part 1 at baseline (mean \pm s.d.) & $10 \pm 5$ & NA \\
PD: MDS-UPDRS Part 2 at baseline (mean \pm s.d.) & $10 \pm 5$ & NA \\
PD: MDS-UPDRS Part 3 at baseline (mean \pm s.d.) & $24 \pm 11$ & $37 \pm 14$ \\
HD: UHDRS-TMS & NA & $44 \pm 3$ \\
HD: Number of CAG repeats & NA & $2(12 \%)$ \\
HD: Neuroleptic use & NA & $8(2)$ \\
HD: UHDRS-Total Functional Capacity & NA & \\
\hline
\end{tabular}




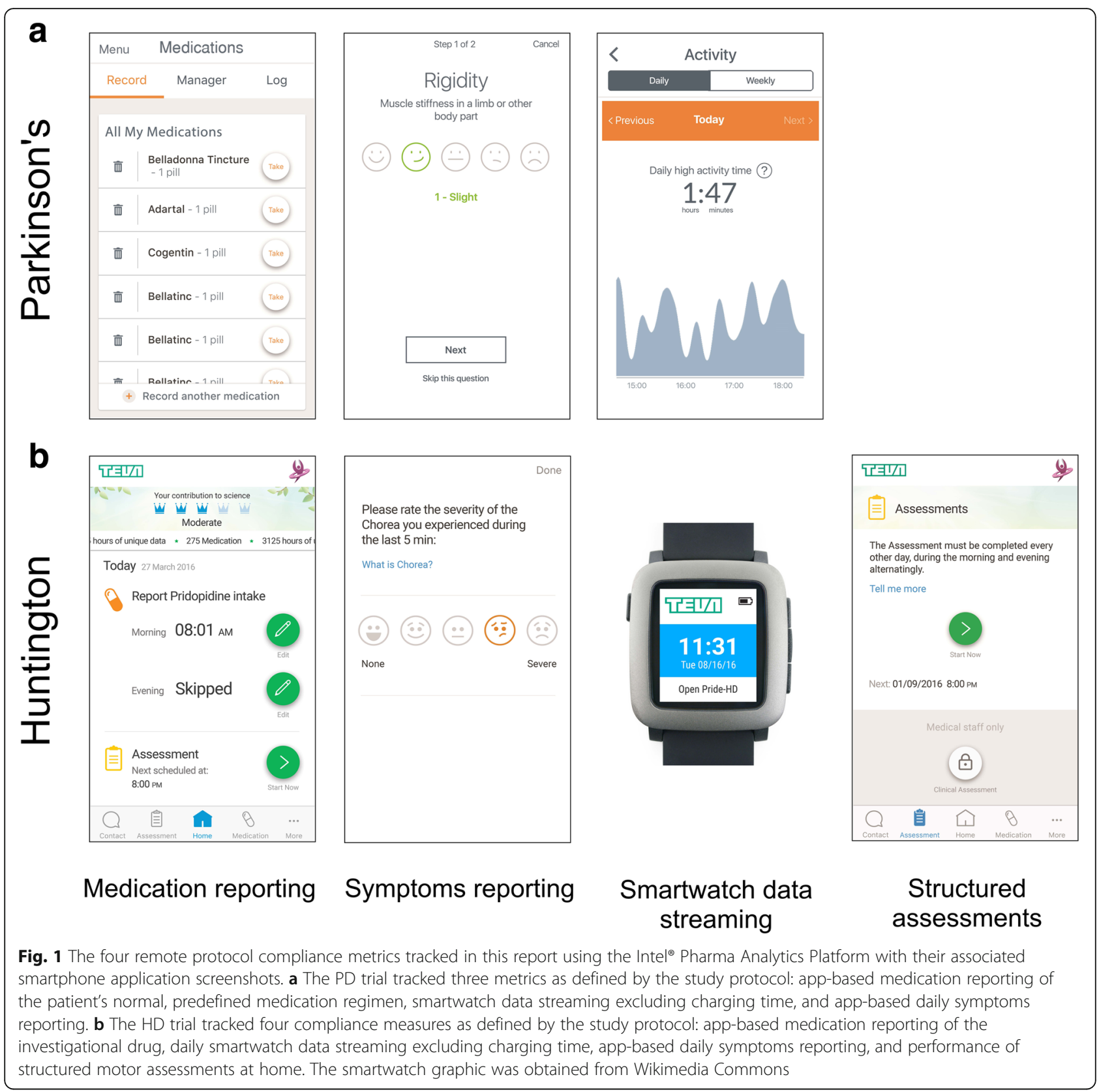

time. Ideally most of this time reflects the hours in which the watch was worn by the patients, although if the watch was turned on, was not charging, and was not being worn those hours would be counted as well. Patients were instructed to follow their normal medication regimen and report on all medication intakes via the dedicated smartphone application throughout the duration of the study. Patients were asked to enter their medication schedule, which was used to prompt daily medication intake reminders on the smartphone. In addition, patients were instructed to wear the smartwatch for a minimum of $12 \mathrm{~h}$ per day for a minimum of 25 days per month. Finally, patients were asked to report their symptom severity three times per day in the dedicated smartphone application. The eight possible symptoms consisted of tremor, dyskinesia, rigidity, bradykinesia, gait problems, balance problems, voice problems, and constipation.

The PD trial incorporated planned support intervention calls and unplanned calls in the case of low compliance. Planned calls were scheduled at time points between the 1,4 , and 5 -month in-clinic visits ( \pm 7 days) to reinforce compliance. Unscheduled telephone calls were made if patients were less compliant than expected with regards to smartwatch data streaming. In addition, the mobile phone application enabled patients to contact technical support via email or phone if needed. 


\section{Huntington disease trial}

The HD trial was an observational sub-study within the larger Open PRIDE-HD open-label, phase 2 trial (ClinicalTrials.gov NCT02494778), and was conducted in 4 countries: the United States, the United Kingdom, Austria, and Germany. The primary eligibility criteria were participation in the larger Open PRIDE-HD study, informed consent, willingness to comply with study requirements, and demonstrated capability to use the smartwatch device and smartphone application. The study recruited 17 patients (instead of the planned total of 60 patients due to early termination of the study) between December 2016 and December 2017, with 9 patients completing the entire study. Study participants were provided an iPhone 6 Plus smartphone and a Pebble smartwatch containing the Intel ${ }^{\circ}$ Pharma Analytics Platform application. Besides two in-clinic visits, the study included a remote component as described below.

Within the trial, we tracked the four protocol-defined metrics related to remote participation: (1) app-based medication reporting, (2) smartwatch data streaming, (3) performance of structured motor assessments at home, and (4) app-based daily symptoms reporting (Fig. 1). In contrast to the PD trial, the HD trial had a home assessments component which did not exist in the former trial. First, patients were asked to take the investigational drug, pridopidine, twice per day per the main study protocol and report intake times using the dedicated smartphone application. Specific instructions were to take the morning dose between 7:00 and 12:00 and the second dose 7 to $10 \mathrm{~h}$ afterwards. Second, patients were instructed to continuously wear the smartwatch on the wrist of the chorea dominant upper-limb, preferably between 9:00 and 21:00. Third, participants were asked to perform a short, structured motor assessment at home every other day, alternating mornings and evenings, comprising two pre-defined tasks: standing still for $30 \mathrm{~s}$ and sitting at rest for $2 \mathrm{~min}$ with arms relaxed. Patients received reminders at the time of assessment (default settings at 9:00 and 18:00, alternating bi-daily) and were able to edit the reminder times. Fourth, patients were asked to report their chorea severity during the last five minutes once a day at a pre-defined time (default reminder at 12:00, time could be edited) using the dedicated smartphone application.

A phone call intervention mechanism was used to improve compliance rates. Support personnel performed monitoring of patient compliance twice a week to ensure fulfillment of two criteria: (1) performance of three structured motor assessments at home over the previous eight days and (2) having a minimal smartwatch data streaming score of three points over the previous three days. The score was calculated by defining a compliant day as having smartwatch data streaming for at least
$90 \%$ of the waking hours (defined as 9:00 to 21:00), and then summing the score of the last three compliant days using the following values for each day: 1 point (for three days ago), 2 points (two days ago), and 4 points (one day ago). Non-compliant days were scored as 0 . Support personal contacted the relevant site for patients that did not fulfill one of the above criteria, which in turn contacted the patient by phone within a few days. In addition, the mobile phone application enabled patients to contact technical support via email or phone if needed.

\section{Compliance metrics calculations}

All metrics were calculated per day and presented smoothed using a moving average with a seven-day sliding window. The duration of smartwatch data streaming was defined by hours of streaming accelerometer data per day, excluding times in which the smartwatch was being charged. To be considered, each streaming hour required at least $90 \%$ of the expected number of records according to the accelerometer sampling rate, which was $50 \mathrm{~Hz}$ in both studies. Medication reporting rates considered both "take" or "skip" as a report. In the PD study, each symptom reporting event could contain between one and eight different symptoms as described above but was considered a single event regardless since it reflected a single interaction with the app.

\section{Results \\ Longitudinal patterns}

We first looked at the aggregated patient compliance rates throughout both studies (Fig. 2). Three compliance metrics were tracked in the PD study and four in the HD study as the latter had an a structured motor assessments at home component which did not exist PD study. We quantify compliance rates as the extent to which the remote study protocol requirements were fulfilled. We observed that compliance rates were markedly distinct during the first few days as compared to the remaining duration of the studies. The duration of smartwatch data streaming was low during the first days for both studies. Initial app-based daily symptoms reporting rates were low and structured motor assessments at home compliance rates were high in the HD study. Given the early study fluctuations, we quantified longitudinal patterns starting at day 14 .

Compliance rates gradually declined in the PD study. Mean daily app-based medication reporting rates dropped $34.2 \%$ from 5.82 to 3.83 (mean during study of 4.85). Mean daily symptom reporting events dropped from 1.61 events to 0.91 , a $43.5 \%$ decline, with the mean of 1.39 being roughly half of the protocol requirement of 3 daily reports. Finally, the daily smartwatch data 


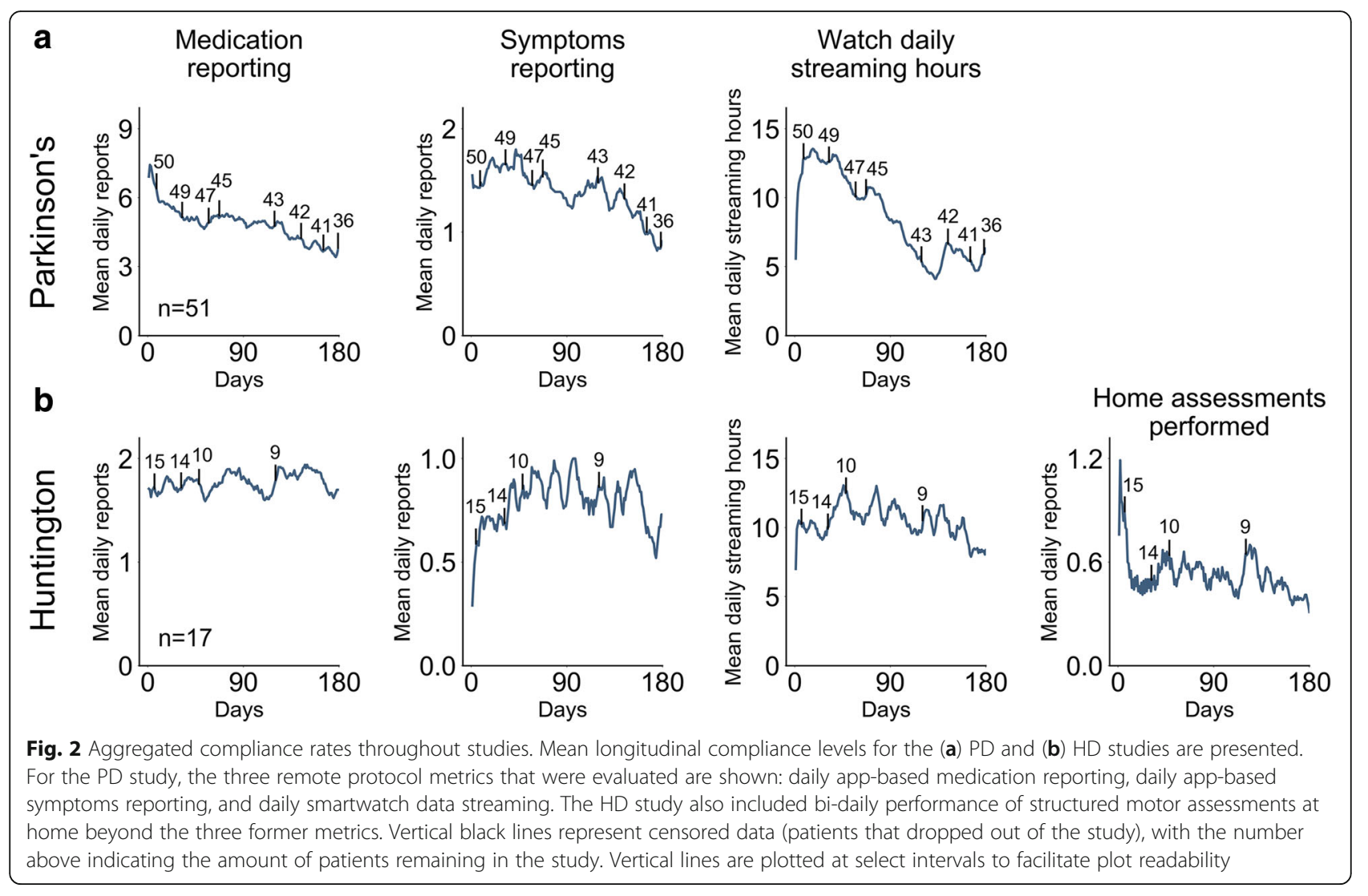

streaming hours declined $52.7 \%$ from $13.32 \mathrm{~h}$ to $6.3 \mathrm{~h}$ (mean during study of $8.6 \mathrm{~h}$ ).

The compliance rates in the HD study varied in their dynamics. Both daily app-based medication reporting and symptoms reporting remained relatively constant. For medication reporting, values decreased only from 1.75 to 1.7 daily reports ( $2.86 \%$ drop). This is close to the expected 2 daily reports. Mean daily symptoms reporting slightly increased by $1.39 \%$ from 0.72 daily reports on day 14 to 0.73 on the final day of the study (study mean of 0.79 daily reports). In contrast, smartwatch data streaming hours decreased $20.36 \%$ from $10.51 \mathrm{~h}$ to $8.37 \mathrm{~h}$ (mean of $10.45 \mathrm{~h}$ for entire study). Finally, the amount of structured motor assessments at home performed declined $30.37 \%$ from 0.95 every two days to 0.66 every two days (mean during study of 1.04 every two days) (Fig. 2 shows the daily rather than bi-daily values for plotting consistency). In both studies, compliance rates were similar among patients who completed the study and those who terminated early (Additional file 1 Figure S1).

\section{Compliance variation per patient}

An examination of individual compliance rates showed that patients generally had either high or low compliance across all metrics (Fig. 3). This is evident, especially in the PD study, as the compliance metric pairs had similar degrees of positive correlations in individual compliance rate (PD metric pairwise correlations: $0.51,0.57$, and 0.59). The effect is stronger in the PD study, perhaps due to higher patient sample size.

\section{Daily and hourly preferences}

We next studied the impact of daily and hourly preferences with respect to the remote protocols. We found that the impact of weekends and holidays on compliance rates was minimal, with only a slight, statistically nonsignificant, decrease in smartwatch data streaming observed in both trials (HD $p=0.50, \mathrm{PD} p=0.71$, two-tailed Student's T-test) (Fig. 4a). Likewise, there were no differences among gender in both studies (Additional file 2 Figure S2), subject age only had a minor correlation with most compliance metrics (Additional file 3 Figure S3), and baseline disease status had only a small correlation with most metrics as well (Additional file 4 Figure S4). The exception was daily smartwatch data streaming hours in the HD study which had positive correlation with age $(R=0.46)$ and baseline UHDRS-TMS rating $(R=0.49)$ (Additional file 4 Figure S4).

In contrast, there was substantial variation in hourly compliance rates. This was expected given the predefined schedules for medication intake in both studies 


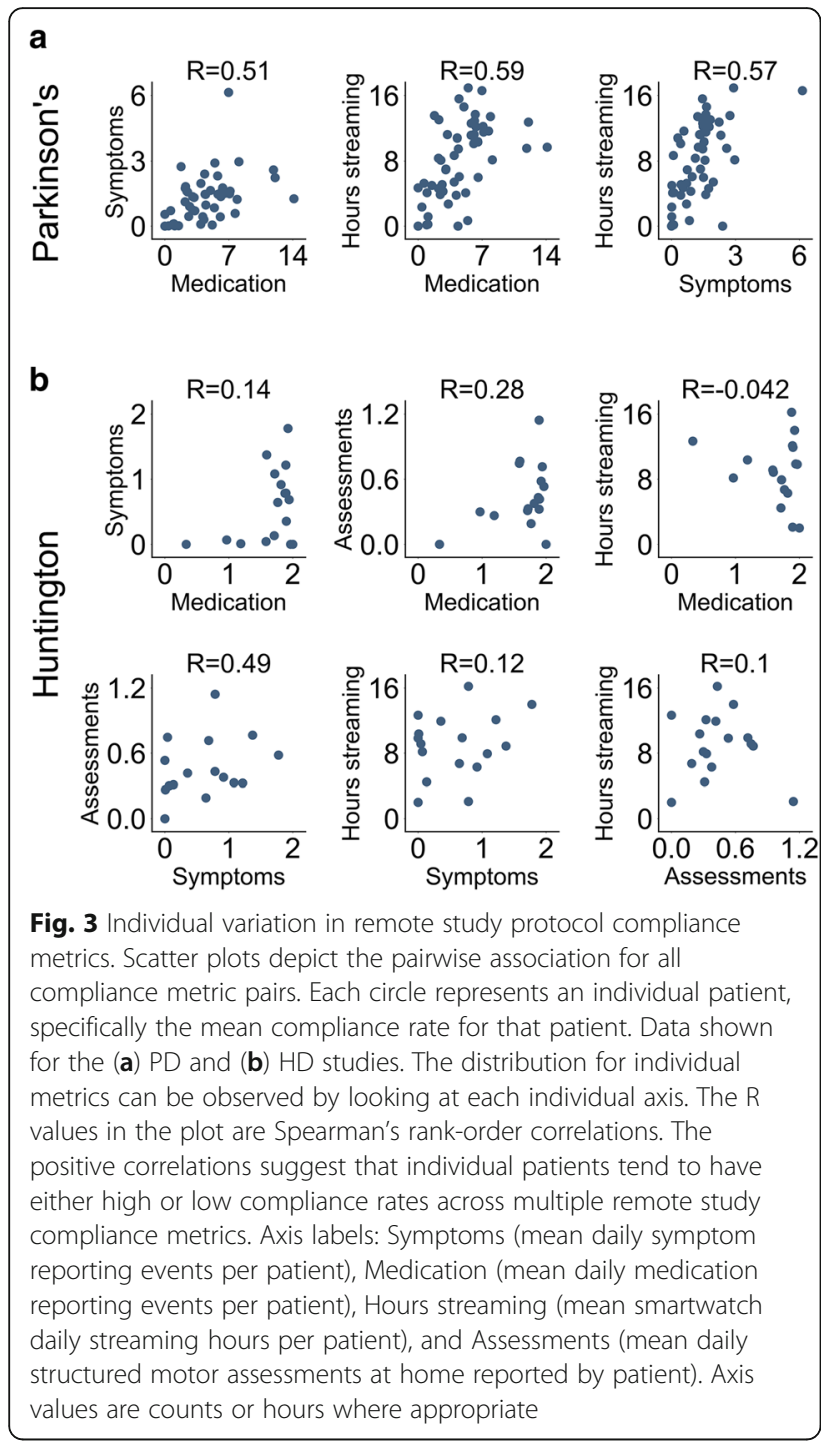

and for app-based symptoms reporting and structured motor assessments at home in the HD study (Fig. 4b). Beyond predefined times, we observed that PD patients preferred reporting on symptoms during the morning hours. Finally, in both studies, smartwatch data streaming rates gradually increased during the morning and peaked at around noon. The level then remained relatively constant until around 20:00 in the HD study, whereas in the PD trial there was a decrease during the afternoon and early evening hours.

\section{Discussion}

Remote monitoring is expected to gradually transform drug development procedures, with assessment of patient compliance using digital monitoring being increasingly useful for smart trial design. The current report includes a relatively long-duration follow-up of movement disorder patients from two unrelated trials

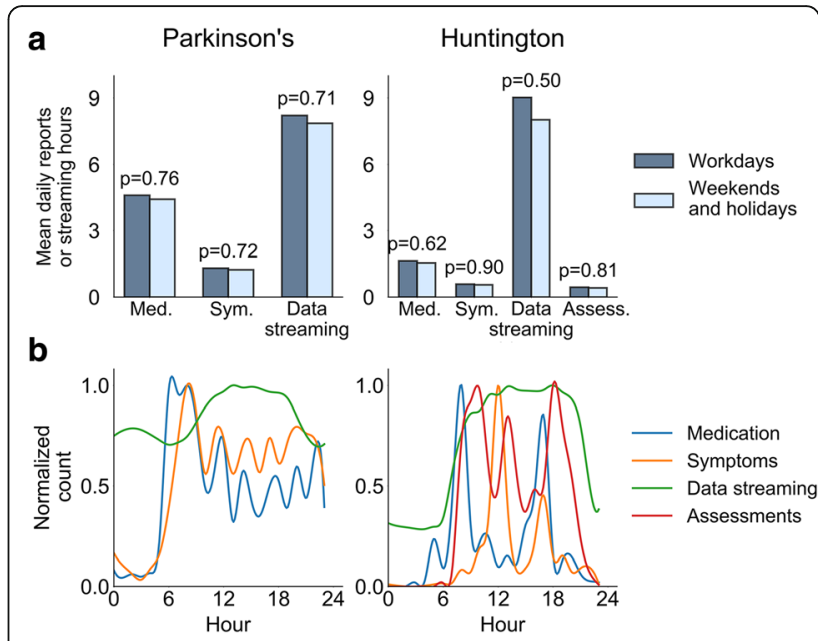

Fig. 4 Hourly and daily compliance patterns. (a) Hourly and (b) daily compliance levels are portrayed for all three remote compliance metrics studied in the PD study and the four metrics studied in the HD study. The hourly patterns may reflect personal preferences and/ or the impact of predefined schedules, as determined by the study protocol, or predefined reminders. Med. - app-based medication reporting, Sym. - app-based symptoms reporting, Data streaming smartwatch data streaming, Assess. - performance of structured motor assessments at home

with respect to four compliance measures that are specific to remote trials.

Several of our findings may assist in the design of similar clinical trials in the future. First, we observed that the variability of the compliance rates at the beginning of the trial are non-indicative of compliance patterns for the vast-majority of the remainder of the trial duration. This is not surprising given the need to adjust to new technology and may need to be considered during trial planning. Second, when given the option, study participants preferred to report their symptoms during the morning hours. Third, defining appropriate eligibility criteria and screening methods appears to be especially important in remote, technology-based trials given that compliant patients tend to exhibit high compliance across the different aspects of the remote trial protocols, rather than to only to a single protocol metric. This is likely influenced by factors such as age, technology savviness, and various additional factors which require further research.

As expected, there were differences in compliance rates over time between the two studies. While smartphone app-based interactions have been shown to increase compliance [16], the observation of gradually decreasing engagement in the PD study was not surprising as decreased compliance over time has been shown in numerous trials, for instance in a trial in which $50 \%$ of patients stopped taking anti-hypertensive drug within one year [24]. One potential reason for the observed 
difference between the trials may be that the HD trial was interventional, perhaps increasing the motivation of patients seeking experimental drug benefits. In addition, the HD trial implemented a higher level of interventional support calls which may have maintained higher compliance levels.

In this work we attempted to understand the adherence of HD and PD patients to remote clinical trial protocols over a multi-month duration. It is important to note that we defined compliance as patient participation levels rather than compliance as strictly defined by the study protocols. While both measures are tightly linked, participation levels are continuous and are thus better suited for understanding patient behavior. In contrast, protocol-defined compliance is often binary due to the definition of thresholds. For example, the PD study protocol defined smartwatch data streaming compliance as streaming data for at least $10 \mathrm{~h}$ per day for 25 days per month.

While HD is an orphan disease with little or perhaps no precedent of studies examining remote compliance, our work is not the first to investigate remote compliance in PD. In the large observational Parkinson@Home study, mean daily smartwatch data streaming durations of $14.8 \mathrm{~h}-16.3 \mathrm{~h}$ were observed for two large cohort studies of 6 weeks (cohort $1, n=304$ ) and 13 weeks (cohort $2, n=649$ ) [13]. As in our PD trial, the researchers observed only a mild reduction in smartwatch data streaming of roughly $25 \%$ from start to finish, which they proposed may be attributed to the passiveness of, or the lack of interaction needed for, data collection. Another study, titled mPower, focused on data collection from thousands of users using home-assessments that leverage smartphone sensors and a corresponding mobile app [14]. In a paper describing the first six months of data collection, the authors observed a rapid, exponential-like drop in average data contribution per patient, which could be due to many factors including the remote recruitment, no interventional treatment, and no support or trial management intervention. Another effort, HopkinsPD collected passive data from the smartphones of PD patients and control subjects and asked for performance of two structured motor assessments at home during the morning hours [15]. While structured assessment compliance relative to protocol was not reported, the authors showed that the abundance of structured assessments at home as well as passive usage of the smartphone remained uniform across all days of the week, similar to our findings.

Patient compliance is a broad topic with many aspects and research challenges. One major challenge is obtaining large patient samples sizes given costs and recruitment challenges, especially in rare diseases. Our HD study had 17 patients, whereas a larger number may potentially impact conclusions. Despite the small sample size, this study provides an example for monitoring patient compliance in long-duration remote studies and can be used in the design, planning and execution of future studies in this patient population. Additionally, factors not addressed in this work such as mobile application user experience and interface design may influence compliance rates. Finally, a deeper analysis of digital trial economics, including device cost, data management and clinic visit savings, can help illustrate the financial considerations in such trials.

\section{Conclusions}

Understanding patterns of patient compliance in remote, technology-based clinical trials requires analysis of data collected using digital technologies. The insights from our work suggest that such remote trials are feasible, even when comprising multiple protocol requirements. Beyond our observations, examination of additional factors that impact compliance such as the impact of support, intervention, alternative application design interfaces, and additional covariates can further influence study design.

In the broader perspective, this report supports the growing trend of using mobile applications and wearable technologies to monitor, prompt, and encourage patient compliance with medication intake and performance of clinical assessments. This effort strengthens the notion that these data have the potential to provide further insight regarding patients' daily lives outside the clinic and potentially evolve into novel endpoints for regulatory purposes.

\section{Additional files}

Additional file 1: Figure S1. Comparison of compliance rates throughout studies between early dropouts and patients that completed the study (DOCX $150 \mathrm{~kb})$

Additional file 2: Figure S2. Compliance patterns by gender for the PD and HD studies (DOCX $87 \mathrm{~kb}$ )

Additional file 3: Figure S3. Compliance patterns by age for the PD and HD studies (DOCX $109 \mathrm{~kb}$ )

Additional file 4: Figure S4. Compliance patterns by baseline disease status for the PD and HD studies (DOCX $103 \mathrm{~kb}$ )

\section{Abbreviations}

CAG: Cytosine Adenine Guanine; CIS-PD: Clinician Input Study in PD patients; HD: Huntington disease; hrs: Hours; Hz: Hertz; IRB: Institutional Review Board; MDS-UPDRS: Movement Disorder Society-Unified Parkinson's Disease Rating Scale; NA: Not available; Open PRIDE-HD: The observational digital health sub-study within Study TV7820-CNS-20016 in HD patients that evaluated pridopidine; PD: Parkinson's disease; s.d: Standard deviation; UHDRS-TMS: Unified Huntington Disease Rating Scale Total Motor Score; UI/UX: User Interface/User Experience

\section{Acknowledgements}

The Clinician Input Study (CIS-PD) was funded by the Michael J. Fox Foundation for Parkinson's Research. We thank the broad PD and HD patient 
communities and especially the specific patients that participated in both studies. We also thank Yonatan Dolan, Shahar Shpigelman, and Chen Admati for insightful discussions during the course of this research, Tatyana Simuni for insightful discussions and medical guidance, Matthew Davis and Nicholas Gross for assistance with statistical analyses, Pippa Loupe for assistance with manuscript editing, and Lena Granovsky for review of sub-study report.

\section{Funding}

The Clinician Input Study (CIS-PD) was funded by the Michael J. Fox Foundation for Parkinson's Research. Teva Pharmaceuticals, who sponsored the observational digital health sub-study in HD patients as a part of a larger HD study (Open PRIDE-HD), was responsible for the design and conduct of the study described in this report. Publication authors affiliated with Teva Pharmaceuticals participated in data analysis, manuscript preparation, and approved final submission.

\section{Availability of data and materials}

The PD dataset is expected to be made public by the Michael J Fox Foundation. The HD patient dataset generated and analyzed during the current study is not publicly available due to privacy reasons but are available from Teva Pharmaceuticals upon reasonable request.

\section{Authors' contributions}

SC and ZW performed data retrieval, statistical analysis of both studies, initial manuscript drafting, and manuscript revision with input from all authors. JJE performed statistical analysis of the CIS-PD data and contributed to manuscript revision. MFG contributed to data interpretation and the drafting and critical revision of the manuscript. IDG was a study physician responsible for HD digital sub-study design, protocol drafting and finalization, medical monitoring, data collection, interpretation of these data, and critical revision of this manuscript. LNP was responsible for the HD digital sub-study protocol drafting and finalization, and contributed to analysis of these data, drafting and critical revision of the manuscript. SF was the co-lead for the HD digital sub-study, responsible for the HD digital sub-study design and execution, including protocol drafting and finalization, technological environment setting and monitoring, data collection, data-scientific analysis, and the interpretation of the analytic outcomes. SP, J-MS, and IG contributed to the HD digital sub-study design and execution, protocol drafting, data interpretation, and critical revision of this manuscript. All authors read and approved the final manuscript.

\section{Ethics approval and consent to participate}

The CIS-PD study protocol and informed consent was approved by the local (Institutional Review Board) IRB committees at each site. The specific four IRBs were: University of Cincinnati Institutional Review Board, University of Alabama at Birmingham Institutional Review Board 01, University of Rochester Research Subjects Review Board, and the Northwestern University Institutional Review Board.

The HD study protocol was approved by relevant site ethics committees and institutional review boards (Western IRB and Chairperson Bert Wilkins, JD, MHA IRB00000533, Puyallup, Washington US; Wake Forest University Health Sciences IRB and Chairperson Sally Bulla, PhD, RN FWA00001732, Winston-Salem, NC US; Georgetown University IRB and Chairperson Charbel Moussa, MD, PhD FWA00001080, Washington DC US; Columbia University IRB and Chairperson Elaine Larson, PhD, RN FWA00002636, New York NY US; Ethikkommission der Medizinischen Universitaet and Chairperson Reinhard Putz, Professor, Dr., Innsbruck Austria; East Midlands - Leicester South Research Ethics Committee and Chairperson John Aldridge, Nottingham UK; Ethik-Kommission der Ärztekammer Westfalen-Lippe und der medizinischen Fakultat der WWU and Chairperson Wolfgang E Berdel, Professor, Dr. med, Rechtsmedizin Germany). Written informed consent was obtained from each patient before any study procedures or assessments were done. These analyses were conducted in accordance with the protocol of the observational sub-study within Study TV7820-CNS-20016 (Open PRIDE-HD) and with the International Conference for Harmonisation Guidelines for Good Clinical Practice, the Declaration of Helsinki, and relevant national and local regulations.

\section{Consent for publication}

Not applicable.

\section{Competing interests}

SC and ZW are Intel employees. MFG, IDG, LNP, SF, IG, SP, and J-MS are or were Teva Pharmaceuticals employees during the course of the study.

\section{Publisher's Note}

Springer Nature remains neutral with regard to jurisdictional claims in published maps and institutional affiliations.

\section{Author details \\ ${ }^{1}$ Advanced Analytics Department, Intel, 94 Em Hamoshavot Road, Petah Tikva, Israel. ${ }^{2}$ Department of Public Health Sciences, Medical University of South Carolina, 135 Cannon St., Suite 303, PO Box 250835, Charleston, SC 29425, USA. ${ }^{3}$ Teva Branded Pharmaceutical Products R\&D, Inc, 41 Moores Rd., Frazer, Petah Tikva, PA 19355, USA. ${ }^{4}$ Guide Pharmaceutical Consulting, LLC, Millstone Township, NJ 08535, USA. ${ }^{5}$ Teva Pharmaceutical Industries Ltd, 12 Hatrufa St, 4250483 Netanya, Israel. ${ }^{6}$ Data Science Institute, Interdisciplinary Center, 1 Kanfei Nesharim St, 4610101 Herzliya, Israel. ${ }^{7}$ CAMP4 Therapeutics, One Kendall Square, Bldg 1400 West, 3rd Floor, Cambridge, MA 02139, USA. ${ }^{8}$ Massachusetts General Hospital, Department of Neurology, Boston, USA. 9Teva Pharmaceuticals International GmbH, Elisabethenstrasse 15, 4051 Basel, Switzerland.}

Received: 2 September 2018 Accepted: 23 November 2018

Published online: 20 December 2018

\section{References}

1. Majumder S, Mondal T, Deen M. Wearable Sensors for Remote Health Monitoring. Sensors. 2017;17:130 Available from: http://www.mdpi.com/ 1424-8220/17/1/130.

2. Coons SJ, Eremenco S, Lundy JJ, O'Donohoe P, O'Gorman H, Malizia W. Capturing patient-reported outcome (PRO) data electronically: the past, present, and promise of ePRO measurement in clinical trials. Patient [internet]. Springer International Publishing. 2015;8:301-9 Available from: https://doi.org/10.1007/s40271-014-0090-z.

3. Hirsch IB, Martinez J, Dorsey ER, Finken G, Fleming A, Gropp C, et al. Incorporating Site-less Clinical Trials Into Drug Development: A Framework for Action. Clin Ther. 2017;39:1064-76 Available from: https://doi.org/10. 1016/j.clinthera.2017.03.018.

4. Streiner DL, Norman GR, Cairney J. Health measurement scales: a practical guide to their development and use. USA: Oxford University Press; 2015

5. Schwartz JE, Burg MM, Shimbo D, Broderick JE, Stone AA, Ishikawa J, et al. Clinic blood pressure underestimates ambulatory blood pressure in an untreated employer-based US population. Circulation. 2016;134:1794-807.

6. Izmailova ES, Wagner JA, Perakslis ED. Wearable devices in clinical trials: hype and hypothesis. Clin Pharmacol Ther. 2018;00:1-11.

7. Espay AJ, Bonato P, Nahab FB, Maetzler W, Dean JM, Klucken J, et al. Technology in Parkinson's disease: challenges and opportunities. Mov Disord. 2016;31:1272-82.

8. Clinical Trials Transformation Initiative. CTTI RECOMMENDATIONS: DEVELOPING NOVEL ENDPOINTS GENERATED BY MOBILE TECHNOLOGY FOR USE IN CLINICAL TRIALS. 2017. Available from: https://www.ctti-clinicaltrials.org/files/ novelendpoints-recs.pdf

9. Del Din S, Godfrey A, Mazzà C, Lord S, Rochester L. Free-living monitoring of Parkinson's disease: lessons from the field. Mov Disord. 2016;31:1293-313.

10. Arora S, Venkataraman V, Zhan A, Donohue S, Biglan KM, Dorsey ER, et al. Detecting and monitoring the symptoms of Parkinson's disease using smartphones: a pilot study. Park Relat Disord [internet]. Elsevier Ltd. 2015;21: 650-3 Available from: https://doi.org/10.1016/j.parkreldis.2015.02.026.

11. Ferreira JJ, Godinho C, Santos AT, Domingos J, Abreu D, Lobo R, et al. Quantitative home-based assessment of Parkinson's symptoms: the SENSE-PARK feasibility and usability study. BMC Neurol [internet]. BMC Neurol. 2015;15:1-7 Available from: https://doi.org/10.1186/s12883-0150343-z.

12. De Lima ALS, Hahn T, Evers LJW, De Vries NM, Cohen E, Afek M, et al. Feasibility of large-scale deployment of multiple wearable sensors in Parkinson's disease. PLoS One. 2017;12:1-15.

13. Bot BM, Suver C, Neto EC, Kellen M, Klein A, Bare C, et al. The mPower study, Parkinson disease mobile data collected using ResearchKit. Sci Data. 2016;3:160011 Available from: http://www.nature.com/articles/sdata201611. 
14. Zhan A, Little MA, Harris DA, Abiola SO, Dorsey ER, Saria S, et al. High Frequency Remote Monitoring of Parkinson's Disease via Smartphone: Platform Overview and Medication Response Detection 2016;1-12. Available from: http://arxiv.org/abs/1601.00960

15. Lakshminarayana R, Wang D, Burn D, Chaudhuri KR, Galtrey C, Guzman NV, et al. Using a smartphone-based self-management platform to support medication adherence and clinical consultation in Parkinson 's disease. Npj Park dis. 2016; 2017:1-8 Available from: https://doi.org/10.1038/s41531-016-0003-z.

16. Silva de Lima AL, Hahn T, de Vries NM, Cohen E, Bataille L, Little MA, et al. Large-Scale Wearable Sensor Deployment in Parkinson's Patients: The Parkinson@Home Study Protocol. JMIR Res Protoc. 2016:5:e172 Available from: http://www.researchprotocols.org/2016/3/e172/.

17. Dinesh $K$, Xiong M, Adams J, Dorsey R, Sharma G. Signal analysis for detecting motor symptoms in Parkinson's and Huntington's disease using multiple body-affixed sensors: A pilot study. Signal Process Work (WNYISPW), 2016 IEEE West New York Image. 2016:1-5.

18. Adams JL, Dinesh K, Xiong M, Tarolli CG, Sharma S, Sheth N, et al. Multiple wearable sensors in Parkinson and Huntington disease individuals: a pilot study in clinic and at home. Digit Biomarkers Karger Publishers. 2017;1:52-63.

19. Andrzejewski KL, Dowling AV, Stamler D, Felong TJ, Harris DA, Wong C, et al. Wearable sensors in Huntington disease: a pilot study. J Huntingtons Dis IOS Press. 2016:5:199-206.

20. Baig MM, GholamHosseini H, Mogeem AA, Mirza F, Lindén M. A Systematic Review of Wearable Patient Monitoring Systems - Current Challenges and Opportunities for Clinical Adoption. J Med Syst. 2017:41.

21. Osterberg L, Blaschke T. Koop - C Everett. Adherence to medication. N Engl J Med. 2005;353:487-97.

22. Cohen S, Bataille LR, Martig AK. Enabling breakthroughs in Parkinson's disease with wearable technologies and big data analytics. mHealth. 2016;2: 20 Available from: http://mhealth.amegroups.com/article/view/10369/10988

23. Daeschler M, Elm J, Klintworth E, Afek M, Lazar S, Simuni T. Clinician-Input Study (CIS-PD): how the Fox Wearable Companion Application can influence treatment and care in Parkinson\{textquoteright\}s disease (P3.048). Neurology. Wolters Kluwer Health, Inc. on behalf of the American Academy of Neurology. 2018:90 Available from: http://n.neurology.org/content/90/15_Supplement/P3.048.

24. Vrijens B, Vincze G, Kristanto P, Urquhart J, Burnier M. Adherence to prescribed antihypertensive drug treatments: longitudinal study of electronically compiled dosing histories. BMJ. 2008;336:1114-7.

Ready to submit your research? Choose BMC and benefit from:

- fast, convenient online submission

- thorough peer review by experienced researchers in your field

- rapid publication on acceptance

- support for research data, including large and complex data types

- gold Open Access which fosters wider collaboration and increased citations

- maximum visibility for your research: over $100 \mathrm{M}$ website views per year

At $\mathrm{BMC}$, research is always in progress.

Learn more biomedcentral.com/submissions 